\title{
GRAPPA 2018 Project Report
}

\author{
Niti Goel (i), Oliver FitzGerald (iD, Dafna D. Gladman (D), Philip S. Helliwell (D), \\ Arthur Kavanaugh, Walter P. Maksymowych ${ }^{\mathbb{D}}$, Philip J. Mease ${ }^{\mathbb{D}}$, Christopher T. Ritchlin (D), \\ and Laura C. Coates (i)
}

\begin{abstract}
At the 2018 annual meeting of the Group for Research and Assessment of Psoriasis and Psoriatic Arthritis (GRAPPA), members received updates on several ongoing efforts. Among them were updates on GRAPPA's patient research partners; educational initiatives; research efforts, including the trainee symposium, pilot research grants, and Collaborative Research Network; treatment recommendations; and additional efforts related to advancing the understanding of disease aspects. (J Rheumatol Suppl. 2019 June;95:54-7; doi:10.3899/jrheum.190121)
\end{abstract}

Key Indexing Terms:

PSORIASIS PSORIATIC ARTHRITIS EDUCATION RESEARCH GRAPPA

Members of the Group for Research and Assessment of Psoriasis and Psoriatic Arthritis (GRAPPA) continue to pursue the core objectives of GRAPPA's mission, specifically, to provide education, identify research assessment

From Kezar Life Sciences, San Francisco, California; Duke University School of Medicine, Durham, North Carolina, USA; Department of Rheumatology, St. Vincent's University Hospital and Conway Institute for Biomolecular Research, University College Dublin, Dublin, Ireland; University of Toronto; Krembil Research Institute, Psoriatic Arthritis Program, University Health Network, Toronto Western Hospital, Toronto, Ontario, Canada; UK National Institute for Health Research (NIHR), Institute of Rheumatic and Musculoskeletal Medicine, University of Leeds, Leeds; Bradford Hospitals UK National Health Service (NHS) Foundation Trust, Bradford, UK; University of California at San Diego, La Jolla, California, USA; University of Alberta, Edmonton, Alberta, Canada; Rheumatology Research, Swedish Medical Center; University of Washington School of Medicine, Seattle, Washington, USA; Division of Allergy, Immunology, and Rheumatology, University of Rochester Medical Center, Rochester, New York, USA; Department of Orthopaedics, Rheumatology, and Musculoskeletal Sciences, University of Oxford, Oxford, UK.

As part of the supplement series GRAPPA 2018, this report was reviewed internally and approved by the Guest Editors for integrity, accuracy, and consistency with scientific and ethical standards.

N. Goel, MD, Patient Research Partner, Chief Medical Officer, Kezar Life Sciences, and Adjunct Assistant Professor, Duke University School of Medicine; O. FitzGerald, MD, FRCP (UK), Newman Clinical Research Professor, Department of Rheumatology, St. Vincent's University Hospital, and Conway Institute for Biomolecular Research, University College Dublin; D.D. Gladman, MD, FRCPC, Professor of Medicine, University of Toronto, Senior Scientist, and Krembil Research Institute, Director, Psoriatic Arthritis Program, University Health Network, Toronto Western Hospital; P.S. Helliwell, DM, PhD, FRCP, NIHR, Institute of Rheumatic and Musculoskeletal Medicine, University of Leeds, and Bradford Hospitals NHS Foundation Trust; A. Kavanaugh, MD, Professor of Medicine, University of California at San Diego; W.P. Maksymowych, MB, ChB, FRCPC, Professor of Medicine, University of Alberta; P.J. Mease, $M D$, Rheumatology Research, Swedish Medical Center, and University of Washington School of Medicine; C.T. Ritchlin, MD, MPH, Professor of Medicine, Division of Allergy, Immunology, and Rheumatology, University of Rochester Medical Center; L.C. Coates, MB ChB, PhD, Department of Orthopaedics, Rheumatology, and Musculoskeletal Sciences, University of Oxford.

Address correspondence to Dr. N. Goel, 4000 Shoreline Court, Ste. 300, South San Francisco, California 94080, USA.

E-mail:agwngw1@gmail.com tools, and pursue research in disease pathophysiology. At the 2018 annual GRAPPA meeting, members received updates on GRAPPA's patient research partners (PRP); educational initiatives; research efforts, including the trainee symposium, pilot research grants, and Collaborative Research Network $(\mathrm{CRN})$; treatment recommendations; and additional efforts related to advancing the understanding of disease aspects such as the axial project, early psoriatic arthritis (PsA) systematic literature review, and imaging.

\section{Patient Research Partners}

It is increasingly recognized that it is important to have patients partner with researchers in efforts related to medical research and education to improve patient outcomes. With that aim, GRAPPA has incorporated PRP into the organization to advance its research and educational objectives. As a subgroup within the GRAPPA organization, PRP have developed their own processes, finalized their policies document, and held their first election for a PRP Chair-elect during 2018. The PRP policies document has been shared upon request to assist with integrating PRP into other similar organizations.

The 2018 annual GRAPPA meeting was the sixth at which PRP have been present. There were 11 PRP who attended (including 1 new member) from North America, South America, Europe, and Asia. PRP were active participants in the meeting's individual sessions and the breakout sessions, with some PRP acting as moderators and/or rapporteurs.

During 2018, the PRP were integrated into several ongoing GRAPPA activities, including the development of the Outcome Measures in Rheumatology (OMERACT)-GRAPPAPsA Core Outcomes Set, the PsA Treatment Guidelines slide deck, the GRAPPA mobile application for Apple and Android devices, the patient self-management brochure, and the GRAPPA CRN.

\section{Education Committee}

The GRAPPA education committee is co-chaired by Philip 
J. Mease and Amit Garg. As a core objective of GRAPPA's mission, GRAPPA members around the world provide psoriasis and PsA education to many people, including other healthcare professionals (physicians, nurses, physician assistants, medical students, residents, and fellows), patients and their families, and healthcare agencies, payor groups, and pharmaceutical employees. The multifaceted approach to educational initiatives includes both continuing medical education (CME) and non-CME comprising live lectures, hands-on patient workshops, Webcasts, audioconferences, online reading material, slides, and videos, as well as textbook chapters, brochures for patient-service leagues, and articles for publication in medical journals.

Owing to GRAPPA's global reach, education occurs in many languages, sometimes aided by simultaneous translators. Although much of this activity is conducted by GRAPPA alone, GRAPPA performs many of these efforts in collaboration with other organizations such as the National Psoriasis Foundation, the International Federation of Psoriasis Associations, the Arthritis Foundation, the American College of Rheumatology (ACR), the European League Against Rheumatism (EULAR), the Pan American League Against Rheumatism, the Asian Pacific League Against Rheumatism (APLAR), the Assessment of Spondyloarthritis international Society (ASAS), and the Spondyloarthritis Assessment and Treatment Network (SPARTAN).

In 2017 and 2018, an ongoing collaboration between GRAPPA and SPARTAN yielded several 2-h, half-day, and full-day symposia on PsA and spondyloarthritis that were predominantly conducted in the United States. A GRAPPA rheumatology-dermatology PsA-psoriasis symposium format is being renewed and will offer a similar variety of options. The longer sessions include breakout sessions to perform patient assessments. The meetings, especially the shorter sessions, are designed to be embedded into society meetings, but all can be conducted as standalone meetings as well. The meetings can be offered as CME or non-CME. In each of these settings, junior and regionally derived faculty teach alongside senior GRAPPA faculty members. These symposia are supported by unrestricted educational grants from several pharmaceutical sponsors.

In addition, GRAPPA has continued to collaborate with SPARTAN and ASAS to conduct a well-attended symposium at the ACR meeting, which took place in San Diego, California, in 2017, and a similar symposium is planned at the ACR meeting in Chicago, Illinois, in 2018. European symposia have been conducted in cities such as Leeds, London, Milan, Bern, Geneva, Stockholm, Sochi, and Moscow. In other parts of the world, GRAPPA has conducted standalone education or partnered with local groups to provide education in India; Tel Aviv; Seoul; Rio de Janeiro, Fortaleza, and Porto Alegre, Brazil; Panama City; and Tokyo. A symposium was also conducted adjacent to APLAR in Taiwan in 2018. Members of GRAPPA look forward to other opportunities to bring PsA education to additional audiences with the PRIME Inc. collaboration and in partnership with PsA Clinics Multicenter Advancement Network.

To aid with lectures, GRAPPA has developed an educational slide library for speakers to use that recently has been revised; it addresses several aspects of psoriatic disease. In addition, GRAPPA members have developed online videos that teach students, residents, fellows, and clinical trial investigators how to properly examine skin, nails, joints, spine, and entheses.

The GRAPPA smartphone application (app) was launched in 2018 and is available to download and use for free on both Apple and Android devices. The app is designed to assist healthcare professionals in clinical practice. The app includes a module to calculate the Psoriasis Area and Severity Index (including pictures that allow the user to gauge severity of the features of psoriasis) and body surface area; a module that allows patients to complete the PsA Impact of Disease (PsAID) questionnaire and that calculates and presents its results to the physician and patient; a module to assess treatto-target goals in PsA that calculates minimal disease activity; and a module to screen psoriasis patients for PsA using the Psoriasis Epidemiology Screening Tool Questionnaire. The app will not record any data internally, to avoid any data protection issues. It also includes reference information for each of the tools and information about GRAPPA.

The PsAID questionnaire module is available in 13 languages: English, Arabic, simplified Chinese, traditional Chinese, French, German, Hebrew, Italian, Japanese, Portuguese, Brazilian Portuguese, Spanish, and Russian. If demand exists, additional languages could be added in future versions. GRAPPA is tracking the number of downloads of the app by country and will use this information to help prioritize which new languages should be added in possible future versions of the PsAID module.

As GRAPPA is increasingly known for its quality in educational endeavors, it was determined that further clarification regarding what qualifies as a GRAPPA-sanctioned educational symposium is needed. A document is planned to define criteria for obtaining GRAPPA endorsement for a symposium. It was agreed that the best practice would be to have GRAPPA review the proposal for an educational program in advance and that the educational program must gain GRAPPA's permission to attach GRAPPA's name to the activity.

\section{Axial Project}

Dafna D. Gladman presented the newly initiated axial project. It is recognized that, while isolated spondylitis is rare among patients with PsA, many patients with PsA have axial disease that is concurrent with peripheral arthritis. On the other hand, psoriasis is observed in 1 in 10 patients with ankylosing spondylitis (AS). It remains unclear whether axial PsA and AS (with or without psoriasis) are different clinical

Personal non-commercial use only. The Journal of Rheumatology Copyright @ 2019 . All rights reserved. 
presentations of the same disease or whether they are separate diseases that have overlapping features. Further, axial disease in PsA has been suboptimally addressed in clinical trials as it has been evaluated only symptomatically without imaging confirmation of axial disease. GRAPPA and ASAS members plan to form a steering committee to define axial PsA; a systematic literature review related to the comparisons between axial PsA and AS has already been completed ${ }^{1}$.

\section{Research Committee}

The Research Committee, under the leadership of Christopher T. Ritchlin, April Armstrong, and Oliver FitzGerald, released a request for applications from trainees and junior investigators for pilot projects in psoriasis and PsA. In 2018, GRAPPA received 20 applications from 11 countries and funded 3 proposals at $\$ 25,000$ each. These proposals were awarded to (1) Graham J. Chapman (UK), Is toe dactylitis in PsA related to trauma caused by plantar shear stress? A case control pilot study; (2) Kim Wervers and Hannah den Braanker (the Netherlands), Response to methotrexate and a quest for personalized medicine in PsA; and (3) Fardina Malik (USA), Gut microbiota as predictor and modulator of methotrexate response in early PsA. The Research Committee will send a new request for applications in early 2019 with additional information and details to be provided at that time.

Additionally, last year's research awardees presented results from their work over the last year. Conor Magee (Ireland) discussed his project, Biomarkers of Progression to PsA in Patients with Psoriasis, and stated that, to date, 190 patients with psoriasis and 100 patients with PsA have been enrolled. The goal of the project is to develop a "bio-profile" based on clinical, genetic, and proteomic biomarkers that is capable of predicting the development of PsA in patients with psoriasis. Patients were categorized at baseline based on musculoskeletal (MSK) signs and symptoms, and these patients are being followed longitudinally to examine which features are predictive of the development of PsA. To date, HLA typing has been performed on almost half of the recruited patients, with the other patients' typing ongoing. A targeted proteomics approach is being used to identify differences in proteins between patients with psoriasis and no MSK features and those with established PsA. Proteins found to be different between these 2 groups will then be used to evaluate prospectively for the development of PsA in the remaining patients with psoriasis.

Margot Van Mechelen (Belgium) discussed how psoriasis and inflammatory bowel disease mouse models align with biomechanical stress to trigger mild joint inflammation. Increasing evidence supports the hypothesis that biomechanical stress, together with inflammatory triggers from distant sites such as the skin or the intestine, can contribute to the onset of PsA, introducing the concept of a Koebner phenomenon at the joint level. This was further investigated by combining a protocol of forced exercise in mice with locally induced cutaneous or intestinal inflammation. This local inflammation triggered a systemic response with inflammation-associated bone loss and discrete signs of joint disease. Forced exercise increased the degree of synovitis and enthesitis in this setting.

Julia Manasson (USA) discussed her project, which is evaluating the effect of biologic therapy on gut bacterial and fungal microbiota pre- and post-tumor necrosis factor inhibitor and interleukin 17 (IL-17) inhibitor therapy. She postulated that gut microbial dysbiosis that is shown to occur in response to IL-17 inhibitor therapy may drive potential gut inflammation. She plans to investigate downstream effects of metagenomic metabolomics and proinflammatory cytokine analyses.

The planned GRAPPA post-meeting for the CRN was also discussed. The presentations included one from keynote speaker Vivian Bykerk, who serves as principal investigator of the Accelerated Medicines Partnership (AMP), a translational research project across numerous sites in the United States. It is funded by the US National Institutes of Health. Another presentation was on pilot projects covering Biomarkers for Joint Damage in PsA, early-disease identification, and detection of treatment response in psoriasis and PsA. In addition, breakout sessions were held that addressed goals, governance, SWOT (strengths, weaknesses, opportunities, threats) analysis, target customers, patient participation, strategic plan, operations, and funding.

Interactions with the Innovative Medicines Initiative (IMI) and with representatives of the pharmaceutical industry were also discussed at the annual meeting in relation to obtaining sustainable funding for the CRN, with additional interactions planned. Because the IMI represents a European Union (EU)-based funding opportunity, it has been decided to register GRAPPA in the EU with the Netherlands as the chosen country following detailed evaluation. Next steps are to retain counsel to assist in the process.

\section{Magnetic Resonance Imaging (MRI)}

Walter P. Maksymowych announced that because assessment of disease activity in PsA lacks objective tools, especially for the assessment of large weight-bearing joints that may disproportionately affect disability, the GRAPPA MRI Working Group has been developing whole-body MRI methodology for the objective assessment of inflammation in synovium, tendon, and bone, together with large joint-scoring methodologies for the assessment of inflammation in the hip and knee. A preliminary scoring method has been developed for whole-body MRI that assesses synovitis and osteitis in 83 peripheral joints and 33 entheses (0-3 grading scheme per joint or enthesis). Images can be put on a Web-based interface, and the reader can evaluate the scan online. Moderate reliability was demonstrated in a preliminary exercise with 8 patients assessed by 14 readers. Work is

Personal non-commercial use only. The Journal of Rheumatology Copyright $@$ 2019. All rights reserved 
ongoing to understand the best calibration methods and scoring for various features.

A granular method for scoring bone marrow lesions in the hip has been developed based on the use of software that applies an overlay to segment the femoral head and acetabulum (Hip MRI Inflammatory Scoring System). This allows multiple regions to be scored directly on a Web-based interface of consecutive MRI slices through the joint (scoring range $0-100$ ), dispensing entirely with the use of scoring spreadsheets. Very good to excellent reliability was attained not only for the detection of femoral head and acetabular lesions at a cross-sectional level, but also for the detection of change 8 weeks after intraarticular steroid injection in scans from 90 patients (180 hips) assessed by 8 readers. Discrimination between treatment groups can now be assessed in clinical trials. Similar work is ongoing for reading knee MRI.

\section{Treatment Recommendations Update}

Arthur Kavanaugh said that the GRAPPA Treatment Guidelines are now 3 years old, and efforts have begun to update them ${ }^{2}$. The approach to update them will be similar to that used for the previous guidelines, which used a modified Grading of Recommendations Assessment, Development, and Evaluation (GRADE) process. GRADE uses evidence to answer 2-by-2 PICO (Patient/Population Intervention - Comparison/Comparator - Outcome) questions. Because PsA is heterogeneous and involves many domains, other evidence-based models may also be applied including Strength of Recommendation Taxonomy. A librarian is being identified to conduct the literature search.

Laura C. Coates presented a brief update on the progress of the International League of Associations for Rheumatology (ILAR) project. With funding from ILAR, a team of about 20 physicians from across the world has worked to adapt existing international recommendations from EULAR and GRAPPA on the management of PsA in resource-poor settings using the ADAPTE Collaboration process ${ }^{3}$. In particular, this project focused on Central America, South America, and Africa. The results of this review and the adapted recommendations were presented at EULAR in Amsterdam in June $2018^{4}$ and will be submitted for publication by the end of 2018 .

\section{Early Psoriatic Disease Systematic Literature Review}

Philip S. Helliwell reported on the setup of a systematic literature review project entitled "Non-topical pharmacological treatment of early, untreated (disease modifying antirheumatic drug-naive, systemic therapy-naive) psoriatic disease: a systematic review." Most trials have been limited with a focus on severe disease of the skin or joints, without looking at more comprehensive indices in outcome assessment. The target condition was defined so that clinical studies investigating MSK and/or cutaneous manifestations, or untreated psoriatic disease in its early stage, could be included. Case reports or studies assessing the effects of topical therapies were excluded. The range of outcome measures considered was broad to assess across the spectrum of disease manifestations and to maximize the sensitivity of the search strategy. The resources to be accessed to find the available evidence were chosen to cover a time range from 1946 to June 2018. The search included electronic databases, trial registers, and conference proceedings. The flow diagram presented during the talk highlighted the substantial number of records $(144,299)$ that were identified through electronic database searches and provided the status of the ongoing title and abstract screening activities by 2 individuals, together with a timeline for publication (June 2019).

This paper summarizes GRAPPA's recent work on a number of projects. These projects are part of GRAPPA's core roles to address educational and unmet research needs, to create opportunities for networking within the psoriatic disease community, and to optimize patient care through collaborative care networks and treatment recommendations.

\section{REFERENCES}

1. Feld J, Chandran V, Haroon N, Inman R, Gladman D. Axial disease in psoriatic arthritis and ankylosing spondylitis: a critical comparison. Nat Rev Rheumatol 2018;14:363-71.

2. Coates LC, Kavanaugh A, Mease PJ, Soriano ER, Laura Acosta-Felquer M, Armstrong AW, et al. Group for Research and Assessment of Psoriasis and Psoriatic Arthritis 2015 treatment recommendations for psoriatic arthritis. Arthritis Rheumatol 2016;68:1060-71

3. The ADAPTE Collaboration. The ADAPTE Process: Resource Toolkit for Guideline Adaptation, Version 2.0 2009. [Internet. Accessed February 21, 2019.] Available from www.g-i-n.net/document-store/working-groups-documents/ adaptation/adapte-resource-toolkit-guideline-adaptation-2-0.pdf

4. Elmamoun M, Eraso M, Maharaj A, Chandran V, Coates L. on behalf of ILAR-PsA recommendations group. International league of associations for rheumatology (ILAR) treatment recommendations for psoriatic arthritis in resource-poor countries [abstract]. Ann Rheum Dis 2018;77:203. 